\title{
L'ÉTENDUE DE LA SOCIOLINGUISTIQUE, LES SCIENCES SOCIALES ET LA NÉCESSAIRE RÉFLEXION COMMUNE SUR « LE SOCIAL »
}

James Costa

Éditions de la Maison des sciences de l'homme | «Langage et société »

2018/1 N 163 | pages 171 à 181

ISSN 0181-4095

ISBN 9782735123995

Article disponible en ligne à l'adresse :

https://www.cairn.info/revue-langage-et-societe-2018-1-page-171.htm

\section{Pour citer cet article :}

James Costa, L'étendue de la sociolinguistique, les sciences sociales et la nécessaire réflexion commune sur « le social », Langage et société 2018/1 ( $\mathrm{N}^{\circ} 163$ ), p. 171-181.

DOI 10.3917/ls.163.0171

Distribution électronique Cairn.info pour Éditions de la Maison des sciences de l'homme.

(c) Éditions de la Maison des sciences de l'homme. Tous droits réservés pour tous pays.

La reproduction ou représentation de cet article, notamment par photocopie, n'est autorisée que dans les limites des conditions générales d'utilisation du site ou, le cas échéant, des conditions générales de la licence souscrite par votre établissement. Toute autre reproduction ou représentation, en tout ou partie, sous quelque forme et de quelque manière que ce soit, est interdite sauf accord préalable et écrit de l'éditeur, en dehors des cas prévus par la législation en vigueur en France. Il est précisé que son stockage dans une base de données est également interdit. 


\title{
L'étendue de la sociolinguistique, les sciences sociales et la nécessaire réflexion commune sur «le social»
}

\author{
James Costa \\ UMR LACITO, université Sorbonne Nouvelle \\ james.costadasorbonne-nouvelle.fr
}

Lorsque Dell Hymes (1974 : 206), définit la portée et l'étendue de la sociolinguistique, il indique qu'elle doit nécessairement porter en elle sa propre fin : "The final goal of sociolinguistics, I think, must be to preside over its own liquidation.» Par là, il entendait que sa mission était de rendre la dimension sociale du langage suffisamment évidente pour qu'elle soit intégrée dans toutes les disciplines pour lesquelles la sociolinguistique ne pouvait être qu'un carrefour temporaire : linguistique, sociologie, anthropologie, mais aussi histoire ou géographie, etc.

En 2013, lors de son intervention dans une table ronde au Congrès $\mathrm{du}$ Réseau francophone de sociolingusitique intitulée "Pouvoir, contre-pouvoir et non-pouvoir... de la sociolinguistique», c'est à un tout autre type de liquidation que fait allusion Josiane Boutet, directrice de la revue Langage \& Société (Boutet 2013, communication personnelle du texte de sa présentation). Boutet constate notamment que concernant la sociolinguistique francophone, quarante ans après sa fondation, la discipline s'est fragmentée en une multitude d'objets et d'approches. Une diversification, dit-elle, "qui pourrait être fructueuse et ne pas conduire à un éparpillement rendant cette discipline invisible dans les SHS, si certaines conditions sociales et intellectuelles étaient remplies». Parmi ces conditions : « une université et des universitaires pacifiés, sûrs de leurs 
valeurs et de leurs missions», et «des politiques de recherche claires, pilotées, permettant la mise sur pied de projets de longue haleine».

En d'autres termes, en 2013, si la sociolinguistique avait présidé à sa propre liquidation, c'est plus par éparpillement que par capillarité. En septembre 2017, lors de son discours d'ouverture de la journée d'étude consacrée aux 40 ans de la revue Langage \& Sociétéé, Michel Wieviorka fait d'ailleurs le même constat à propos de l'ensemble des sciences sociales, dont il dit qu'elles sont "émiettées». Il ajoute qu’il faut aujourd'hui restructurer les champs, non pour « reconstruire des dogmatismes mais aider à ce que se reconstruisent des images de plus en plus claires, des grands axes qui vont structurer les débats au sein de notre espace scientifique».

Poser la question en termes de champ, c'est bien entendu poser la question des limites du champ - des limites non pas posées a priori (la sociolinguistique comprend tout ce qui a trait au langage en société) mais vues comme un enjeu, une lutte pour définir les objets légitimes de recherche et la manière de les appréhender (Bourdieu 2013). À mon sens, c'est dans cette démarche que s'inscrivent les deux livres que je vais tenter de discuter et de mettre en rapport dans ce débat : celui de Nikolas Coupland (dir.), Sociolinguistics: Theoretical Debates, (Cambridge, Cambridge University Press, 2016) et celui de Cécile Canut et Patricia von Münchow (dirs), Le langage en sciences humaines et sociales (Limoges, Lambert Lucas, 2015). À leur manière, tous les deux tentent de proposer, sinon d'imposer, une manière de penser le lien entre sociolinguistique et sciences sociales (et non plus, comme ça a pu être le cas par le passé, entre sociolinguistique et linguistique) et de définir les débats centraux, c'est-à-dire légitimes, de la discipline. Face à l'émiettement partout constaté, ce travail est nécessaire, mais il n'est pas inintéressant de réfléchir à la manière dont la constitution d'axes de réflexion légitime est envisagée en France d'une part (dans l'ouvrage de Canut et von Münchow) et de manière internationale de l'autre (dans celui de Coupland).

On aurait pu bien sûr prendre d'autres livres comme base à cette discussion, il n'en manque pas. Pourtant, chacun de ces deux ouvrages annonce dans son titre une ambition programmatique qui rend la comparaison à mon sens pertinente : Le langage en sciences sociales d'une part, et Sociolinguistics: Theoretical Debates de l'autre. Il faut aussi faire

1. La video est disponible en ligne à l'adresse suivante $:<$ https://www.canal-u.tv/video/ fmsh/la_revue_langage_societe_fete_ses_40_ans_1_ouverture_de_la_journee.37815>. 
remarquer que la distinction a priori entre une sociolinguistique francophone et une sociolinguistique anglophone n'est que partiellement pertinente. Pour ne citer qu'eux, Monica Heller et Alexandre Duchêne d'une part, et Alexandra Jaffe d'autre part, tous trois contributeurs au livre de Coupland, participent également largement à la vie d'une sociolinguistique francophone. D'autre part, des auteurs comme Foucault et Bourdieu sont largement cités par des auteurs qui s'expriment en anglais (et pour qui ça n’est pas nécessairement la langue principale en dehors de l'activité scientifique).

Dans cet article de débat, je souhaite interroger les objets et les théories qui sont présentés et promus, c'est-à-dire légitimés, dans ces deux ouvrages qui sont de fait programmatiques. Je voudrais aussi pointer un manque, à savoir la survalorisation de l'aspect "langue» au détriment de l'aspect «social» de la discipline, dont la problématisation et la théorisation semble avoir peu évolué en sociolinguistique au cours des quarante dernières années - au contraire de ce qui a pu se passer en anthropologie sociale ou en sociologie des sciences par exemple.

\section{Sociolinguistique et sciences sociales}

Ce manque semble trouver son origine dans un clivage qui accompagne la naissance même de la discipline, prise, nous dit Lahire dans son article reproduit dans l'ouvrage de Canut et von Münchow (2015 : 21), dans un étau qui opposerait (en apparence au moins) «les sciences dites sociales : anthropologie, histoire, sciences politiques, économie, etc.) d'une part, et la linguistique (et, plus largement, toutes les sciences des productions symboliques : sémiologie, analyses de discours, théories esthétiques, théories de la littérature, etc.) d'autre part, [constituant] un puissant obstacle à la compréhension et des phénomènes dits sociaux et des phénomènes dits linguistiques (symboliques, esthétiques, iconiques, discursifs, textuels, etc.)».

Que ces deux aspects ne représentent que deux faces d'une même réalité, comme l'écrit Lahire, n'empêche en aucun cas que cette tension traverse l'ensemble de la sociolinguistique et favorise son éclatement vers un pôle ou un autre, vers une attention portée à la représentation et le symbolisme (Kohn 2015) ou vers l'action et la pratique. Dans la continuité des travaux de Boutet notamment, c'est en partie vers une réconciliation de ces deux pôles que souhaite aller le livre de Canut et von Münchow, programme poursuivi et affiné par Cécile Canut dans un ouvrage à paraitre (Canut, Danos, Him-Aquilli, \& Panis, à paraître) à travers une mise en rapport plus fine d'une sociolinguistique critique et 
de l'analyse du discours telle que développée à l'origine autour de Michel Pêcheux. Le langage est appréhendé comme un faire, et le sens comme constitué dans et par l'action, reprenant là une tradition philosophique ancienne, de la kabbale (Eco 1994) à certains écrits en philosophie analytique comme ceux d'Ernest Gellner (1959).

L'objectif est bien, me semble-t-il, d'aller vers une anthropologie du langage française, qui puisse parler à la fois aux linguistes d'une part et aux sociologues, anthropologues, géographes, etc. d'autre part. En ce sens, peu importe l'objet sur lequel porte la parole : ce qui est important dans la proposition que formule ce livre, c'est bien l'attention au langage comme non transparent et aux processus de construction du sens en action et dans l'action. Il s'agit de proposer une cohérence au moins méthodologique à la fois pour la sociolinguistique et pour les praticiens des sciences sociales, qui seraient, selon Lahire, découragés justement par l'éparpillement dont font preuve les approches sociales du langage.

Finalement, une simplification du domaine pourrait impliquer une division de ces approches entre une sociolinguistique ou une anthropologie de la parole en action d'une part, et le livre de Canut et von Münchow s'inscrit dans ce cadre, et une sociolinguistique des langues comme institutions, telle qu'elle peut être pratiquée à Montpellier par exemple. Une telle division est sans doute simpliste, les discours sur les langues pouvant parfaitement être analysés selon les méthodologies du premier courant. Certains, comme Robert Lafont, se sont d'ailleurs jadis confrontés aux deux approches, mais on sait quels chemins elles ont suivi de manière séparée. Cette dichotomie a peut-être le mérite d'entériner deux types d'objets très distincts finalement, le langage d'une part, et les langues de l'autre.

\section{Débats théoriques en sociolinguistique}

À l'inverse du livre de Canut et von Münchow, celui de Coupland ne propose rien pour l'avenir de la discipline, il entérine les débats théoriques dominants dans le champ et de fait les légitime comme tels. Il se veut certes plus large que l'ouvrage de Canut et von Münchow en ce sens qu'il prétend faire le point sur les débats les plus importants, mais il reste silencieux en termes de propositions. Organisé en six parties, le livre traite 1 . de la théorisation du sens en société; 2 . du langage, des marchés et de la matérialité; 3 . de l'espace et la mobilité; 4. des questions de pouvoir et de critique sociale; 5 . de l'impact de la discipline; et 6. de l'évolution de la théorie en sociolinguistique. Les thèmes correspondent bien aux grands débats que l'on peut suivre dans les trois revues 
anglophones principales dans lesquelles s'expriment les sociolinguistes, à savoir Language in Society, Journal of Sociolinguistics, et Journal of Linguistic Anthropology. Des media (Androutsopoulos) au translanguaging (Pennycook), de la complexité (Blommaert) à la gouvernementalité (Rampton), des marchés linguistiques (Kelly-Holmes) ou du langage comme ressource (Heller \& Duchêne) à la question de la matérialité des corps (Bucholtz \& Hall), de la variation (Eckert) à la différentiation (Gal) jusqu’à des processus plus sémiotiques (Jaffe ou Silverstein), l'ouvrage de Coupland se pose en un état de la discipline en 2016, année de sa publication.

Dans son introduction, Coupland (2016 : 2) fait également le constat de l'éparpillement de la discipline, tiraillée non seulement entre différentes directions et théories, mais entre différents types de théories. C'est d'ailleurs cet éparpillement qui lui fait définir la sociolinguistique de manière minimaliste comme "the fertile and shifting multi- and interdisciplinary fields of enquiry where language and society come into contact with each other in so many ways" (ibid. : xi), définition à la fois large, vague, et problématique - le langage «entre-t-il en contact» avec la société, ou en est-il partie prenante?

Ce qui ressort de ce découpage c'est une attention particulière portée d'une part à la variation et d'autre part à la critique. La question qui semble sous-tendre l'ensemble du livre reste bien celle du changement sociolinguistique (et linguistique), question qui hante finalement toute la sociolinguistique et une grande partie de l'anthropologie linguistique anglophone depuis leurs origines. Changement linguistique autant que changement social, puisque le changement sociolinguistique est défini par Coupland comme "consequential change over time in language-society relations" (2016 : 433, italiques dans l'original). Ce changement, ajoute Coupland, peut être appréhendé à partir de cinq mots-clefs en $\mathrm{M}$ : marchés, mobilités, modalités (et théorie du langage), media et métacommunication. Ces mots-clefs, et le programme annoncé de la discipline (le changement sociolinguistique, qui permet de distinguer l'universel et l'historiquement contingent) permettent à Coupland d'amorcer une réflexion sur le lien entre théorie sociolinguistique et théorie sociale. Selon lui, la première ne doit pas devenir une simple annexe de la seconde.

La critique, quant à elle, est particulièrement saillante dans l'attention portée aux processus de différentiation, fruit de plus de vingt années de travail à la croisée de l'anthropologie et de la sémiotique autour de Susan Gal et Judith Irvine (à partir de 1995 notamment). Elle est également 
présente, influencée par Marx comme par Bourdieu (1977) dans les chapitres sur le marché qui traduisent ce qu'on pourrait voir comme un tournant économiciste dans la sociolinguistique critique depuis les travaux de Heller, Duchêne ou Kelly-Holmes. Ces travaux portent clairement l'ambition de réfléchir à une économie politique de la langue et du langage (voir en particulier Del Percio, Flubacher \& Duchêne 2016), même si finalement ils insistent davantage sur l'économique que sur le politique.

Les deux volumes ont bien une volonté programmatique, même si elle n'est pas affirmée de la même manière : une jonction méthodologique entre sociolinguistique et analyse de discours pour l'ouvrage de Canut et von Münchow affirmant la nécessaire prise en compte de la matérialité langagière et discursive au-delà des objets étudiés; une série de débats actuels questionnant le langage et la société dans le cas de Coupland, débats choisis en fonction de leur importance selon luimême, de son propre aveu. Les deux livres sont certes très différents et reflètent des généalogies scientifiques bien distinctes, même si certains grands ancêtres comme Bourdieu peuvent sur certains points servir de points de convergence.

\section{Repenser le social?}

Ce qui réunit les deux ouvrages, mais c'est sans doute peu surprenant, c'est l'entrée par le langage ou la langue, et la primauté de la question du langage sur celle du «social» - une forme de logocentrisme en d'autres termes. Ce qui est légitime pour la sociolinguistique, mais qui pour cette raison aboutit à faire, dans une certaine mesure, l'économie d'une réflexion renouvelée sur ce qu' est ce «social», ce "socio-» de la sociolinguistique, même après la perte de son tiret. Ce faisant, la sociolinguistique risque de tomber dans le piège pointé par Bruno Latour :

All the disciplines from geography to anthropology, from accounting to political science, from linguistics to economics, enter the scene as so many of the ways through which the ingredients of the collective are first juxtaposed and then turned into some coherent whole. (Latour 2005 : 257)

En d'autres termes, le poids de certaines traditions de réflexion sur le social lui-même en sociolinguistique pose les mêmes problèmes qu'en économie ou en anthropologie socioculturelle, considérant finalement la langue comme un ingrédient qui, juxtaposé à d'autres, forme un tout plus ou moins cohérent qui serait appelé société. Le social, en d'autres 
termes, serait ce qui reste quand on en a retiré le strictement linguistique ou économique, etc. - d'où la possibilité d'une langue qui «entre en contact» avec la société, comme évoquée par Coupland dans sa préface. Si ce biais est moins marqué dans l'approche de Canut et von Münchow, puisque le langage y fait le social, la réflexion sur la nature du social apparait surtout en filigrane. Que cette question soit présente en filigrane ne signifie pas qu'elle est absente, bien sûr. Comme le rappelle Coupland dans son introduction, il a été largement reproché à Chambers de ne pas s'interroger sur la manière dont "le langage» interagit avec "le social» (2016 : 5, les guillemets sont de Coupland), et à Labov son côté parsonien et orienté vers une sociologie du consensus. D'autres reproches ont pu être formulés à l'égard d'une sociolinguistique de la politesse comme étant sous-tendue par une conception de la société comme résultant de choix rationnels (ibid.). La question du social, de la société, sous-tend donc, implicitement ou explicitement, toute réflexion sociolinguistique. Mais plus largement, la question du langage ne peut pas se dérouler en dehors d'une réflexion sur le social - ou sur le politique d'ailleurs, la réflexion sur ce qui est politique étant finalement absente des deux volumes.

Si la proposition de Canut et von Münchow est davantage méthodologique, celle de Coupland est plus théorique. Les deux visent à rassembler la discipline, à la rendre plus lisible. Mais, puisqu'il s'agit ici de la rubrique "débat», je me risquerai à une observation : le plus important, me semble-t-il, pour toute discipline académique, et ce à toute époque, ne me semble pas seulement résider dans les débats théoriques ou dans les options méthodologiques, mais dans les questions que nous formulons pour identifier et répondre aux enjeux d'une époque. Or ces questions sont absentes des deux ouvrages, surtout dans celui de Coupland; ou bien elles recyclent des questions qui nous viennent des XVIII et $\mathrm{XIX}^{\mathrm{e}}$ siècles. Se priver de reposer la question de ce qu'est le social, et pas seulement de ce qu'est le langage, c'est se priver de s'interroger sur qui, et surtout quoi, entre dans la constitution du social.

Ce faisant, la sociolinguistique repose sur une vision humano-centrée du social, et s'interdit de repenser, face aux conditions actuelles de changement climatique notamment (Latour 2015), la question posée à la naissance des sciences sociales dans les questionnements philosophiques de Rousseau, Hume ou Smith : comment la société tient-elle ensemble, et comment faire en sorte qu'elle tienne le mieux possible? Car c'est bien de cette question, par exemple, que découle la problématisation du marché chez Adam Smith comme mécanisme neutre pour assurer 
la redistribution des ressources en dehors du contrôle de la corruption des élites à l'époque (Herman 2001). C'est aussi de cette recherche de mécanismes neutres, localisés en dehors de la société, que dérive l'idée d'une langue standard neutre (Costa, De Korne \& Lane 2017), une voix de nulle part (Gal \& Woolard 1995). Mais ces réponses ne sont pas universelles, elles découlent de conditions historiques bien particulières, héritières des guerres de religion en Europe au siècle précédent et de la mise en place, pour répondre à ces conflits, du système des États-nations (Toulmin 1990).

Il semble difficile aujourd'hui de faire l'impasse sur les changements climatiques. Intégrer le réchauffement de la planète dans l'équation du social, et avec cette donnée une myriade d'entités non-humaines, c'est aussi penser les mobilités dues au réchauffement, mais c'est surtout repenser le politique et ce qui peut constituer un objet et un sujet politique. Comme l'écrivent Braun et Whatmore (2010 : xiv-xv), les théories politiques modernes, fondées sur la notion de débat public neutre et du langage comme potentiellement purifiable (de ses indexicalités de lieu ou de classe sociale au moins, sinon de genre) pour accéder à cette neutralité sont fondées sur l'idée que les sociétés ne sont composées que d'humains. Cette approche a eu pour conséquence de rejeter tout le non-humain en dehors du domaine du politique pour le reléguer au rang de ressource, «entering political theory only to the extent that it has instrumental value but not in terms of its constitutive power " (ibid.). Il existe actuellement une réflexion fertile sur ce qu'est le politique en anthropologie socioculturelle, qui considère que toute entité peut devenir un sujet politique en tant qu'elle met en lien, ou en réseau, des groupes et des entités qui auparavant étaient indépendantes. Ainsi, en Amérique latine, Tatiana Li (2013) analyse-t-elle une montagne en termes politiques alors qu'elle est la proie des appétits d'une compagnie minière, transformant la montagne en sujet politique reliant divinités indigènes, groupes indigénistes, militants environnementaux, etc. Ces réflexions peuvent-elles avoir un impact sur la manière de penser le langage en société, et le langage tout autant que le social? Curieusement (ou pas), la réflexion la plus avancée sur cette question ne vient pas de l'anthropologie linguistique ou de la sociolinguistique mais de l'anthropologie socioculturelle et de la tentative d'Eduardo Kohn de "provincialiser le langage» pour comprendre comment les êtres, humains et non-humains, communiquent en Amazonie (Kohn 2013). Son livre a été récemment traduit en français sous le titre Comment pensent les forêts : vers une anthropologie au-delà de l'humain. 
Mais si les sociolinguistes se montrent réticents à repenser leurs notions et conceptions du social et de la société, la discipline aurait justement fort à contribuer aux débats actuels ayant lieu au sein de la sociologie des sciences ou dans certains secteurs de l'anthropologie sociale. Car si ces lieux sont fertiles pour repenser la société comme étant à composer, plutôt que pré-existant aux échanges (Latour 2011), la cosmopolitique de Stengers (2007) ou les réflexions sur les collectifs de Latour ou Descola manquent singulièrement de langage. Comment les êtres, humains ou non-humains, impliqués dans ces collectifs communiquent-ils? Par quels moyens sont-ils organisés en collectifs? Si les XVII ${ }^{\mathrm{e}}$ et XVIII ${ }^{e}$ siècles n'ont pu faire l'économie d'une réflexion sur le langage pour résoudre les questions que leurs contextes respectifs posaient, comment le $\mathrm{XXI}^{\mathrm{e}}$ siècle pourrait-il se passer d'un véritable questionnement qui ne sépare plus nature, société et langage (Bauman \& Briggs 2003) mais les réunisse à nouveau, en interrogeant particulièrement la question du langage? Quel type de conception du langage une cosmopolitique nécessite-t-elle? Il y a là un espace de discussion dans lequel une sociolinguistique ou une anthropologie du langage qui chercherait à dépasser l'opposition entre pratique et analyse de la construction du sens aurait toute la place de s'engouffrer, permettant de participer à une réflexion sur ce qui constitue une société au Xxi ${ }^{\mathrm{e}}$ siècle et sur ce qu'est ou devrait être un sujet et une action politiques.

\section{Références bibliographiques}

Bauman R. \& Briggs C. L. (2003), Voices of Modernity: Language Ideologies and the Politics of Inequality, Cambridge, Cambridge University Press.

Bourdieu P. (2013), "Séminaire sur le concept de champ, 1972-1975», Actes de la recherche en sciences sociales 200(5), p. 5-37.

Bourdieu P. (1977), «L'économie des échanges linguistiques», Langue française 34, p. 17-34. 
Braun B. \& Whatmore S. J. (2010), "The stuff of politics: An introduction", in Braun B. \& Whatmore S. J. (dirs), Political Matter: Technoscience, Democracy, and Public Life, Minneapolis, University of Minnesota Press, p. ix-xl.

Canut C., Danos F., Him-Aquilli M. \& Panis C. (à paraître), Le langage, une pratique sociale. Élements d'une sociolinguistique engagée.

Canut C. \& von Münchow P. (dirs) (2015), Le langage en sciences humaines et sociales, Limoges, Lambert Lucas.

Costa J., De Korne H. \& Lane P. (2017), "Introduction. Standardising minority languages: Reinventing peripheral languages in the $21^{\text {st }}$ century?", in Lane P., Costa J. \& De Korne H. (dirs), Standardizing Minority Languages: Competing Ideologies of Authority and Authenticity in the Global Periphery, London, Routledge, p. 1-23.

Coupland N. (dir.) (2016), Sociolinguistics: Theoretical Debates, Cambridge, Cambridge University Press.

Del Percio A., Flubacher M., \& Duchêne A. (2016), "Language and political economy", in Garcia O., Flores N. \& Spotti M. (dirs), Oxford Handbook of Language in Society, Oxford, Oxford University Press, p. $55-75$.

Eco U. (1994), La recherche de la langue parfaite dans la culture européenne, Paris, Seuil.

Gal S. \& Irvine J. T. (1995), "The boundaries of languages and disciplines: How ideologies construct difference", Social Research 62(4), p. 9671001.

Gal S. \& Woolard K. A. (1995), "Constructing languages and publics: Authority and representation”, Pragmatics 5(2), p. 129-138.

Gellner E. (1959), Words and Things, Boston, Beacon Press.

Herman A. (2001), The Scottish Enlightenment: The Scots' Invention of the Modern World, London, Harper Perennial.

Hymes D. (1974), Foundations in Sociolinguistics, Philadelphia, University of Pennsylvania Press.

Kohn E. (2015), "Anthropology of Ontologies", Annual Review of Anthropology 44(1), p. 311-327. En ligne : <http://doi.org/10.1146/annurev- 
anthro-102214-014127>.

Kohn E. (2013), How Forests Think: Toward an Anthropology Beyond the Human, Berkeley/Los Angeles, University of California Press.

Latour B. (2015), Face à Gaïa. Huit conférences sur le nouveau régime climatique, Paris, La Découverte.

Latour B. (2011), «Il n'y a pas de monde commun : il faut le composer», Multitudes 45, p. 38-41. En ligne : <http://doi.org/10.3917/ mult.045.0038>.

Latour B. (2005), Reassembling the Social: An introduction to Actor-Network Theory, Oxford, Oxford University Press.

Li T. (2013), "Relating divergent worlds: Mines, aquifers and sacred mountains in Peru", Anthropologica 55(2), p. 399-411.

Stengers I. (2007), «La proposition cosmopolitique», dans Lolive J. \& Soubeyran O. (dirs), L'émergence des cosmopolitiques, Paris, La Découverte, p. $45-68$.

Toulmin S. E. (1990), Cosmopolis: The Hidden Agenda of Modernity, Chicago, University of Chicago Press. 